\title{
Rapid and efficient synthesis of the pentapeptide of elastin protein and peptides containing highly hindered $\alpha, \alpha$-dialkyl amino acids employing Fmoc-amino acid chlorides under microwave irradiation in the solution phase
}

\author{
Subramanyam J. Tantry, R. V. Ramana Rao, and V. V. Suresh Babu \\ Department of Studies in Chemistry, Central College Campus, Dr. B. R. Ambedkar Veedhi, \\ Bangalore University, Bangalore-560 001, India \\ E-mail: hariccb@,rediffmail.com
}

\begin{abstract}
A rapid and efficient synthesis of peptides in solution employing Fmoc-amino acid chlorides under microwave irradiation is described. A comparison study of the microwave assisted method with those of conventional peptide synthesis using acid chlorides and various coupling additives has been performed. It has been found that, in general, the formation of a peptide bond, employing Fmoc-amino acid chloride and zinc dust or TBDMS-OBt under microwave irradiation is complete in 30-45 seconds with $90 \%$ yield of pure isolated peptide. Employing zinc dust as a coupling additive, the synthesis of several dipeptides, the pentapeptide fragment FmocVal-Pro-Gly-Val-Gly-OBzl, of elastin and the difficult highly hindered couplings of $\alpha, \alpha-$ dialkylamino acids are reported.
\end{abstract}

Keywords: Fmoc-amino acid chlorides, zinc dust, TBDMS-OBt, elastin-pentapeptide, $\alpha, \alpha-$ dialkylamino acids

\section{Introduction}

Synthesis of peptides employing the shelf-stable 9-fluorenylmethoxycarbonyl (Fmoc) amino acid chlorides makes couplings of even very hindered or weakly nucleophilic systems possible where other methods give only poor results. ${ }^{1,2}$ The trapping of the liberated hydrogen chloride during coupling has been accomplished by using a two-phase system with a mild inorganic base in the aqueous layer. ${ }^{3}$ Improvements in the coupling in homogeneous system by the use of coupling additives like $\mathrm{AgCN}$, ${ }^{4,5}$ potassium salt of 1-hydroxybenzotriazole ${ }^{6,7}$ and 1-hydroxy-7-azabenzotriazole $^{8}$ along with Fmoc-amino acid chloride circumvented the formation of oxazol$5(4 \mathrm{H})$-one but also resulted in elimination of the premature deblocking of Fmoc group during 
prolonged coupling reactions. 1-( $t$-Butyldimethylsilyloxy)benzotriazole (TBDMS-OBt) ${ }^{9}$ has been found to be advantageous due to its solubility in $\mathrm{CH}_{2} \mathrm{Cl}_{2} / \mathrm{CHCl}_{3}$ and the separation of the side product TBDMS-Cl is also simple. $\mathrm{Zinc}^{10}$ and indium ${ }^{11}$ mediated coupling is an alternative reaction pathway for the mediation of coupling by the acid chloride method. Some of the advancements led to complete coupling in about $30 \mathrm{~min}$. The need for further acceleration of the synthesis of peptides with rapid, efficient and high yield coupling is addressed in this communication, demonstrating yet another utility of microwave irradiation in organic synthesis. $^{12-14}$

The utility of microwave irradiation for the synthesis of several building blocks ${ }^{15-19}$ useful in the synthesis of peptides and peptidomimetics like benzyl esters, conversion of Fmoc-/Boc- $\alpha$ aminodiazoketones to $\beta$-amino acids by Wolff rearrangement, Fmoc-/Z-/Boc- $\alpha$-amino acids to Fmoc-/Z-/Boc-5-oxazolidinones and Fmoc- $\alpha$-amino acid azides to the corresponding isocyanates has been demonstrated by us. The solid phase peptide synthesis employing Fmoc-amino acids and symmetric anhydride or preformed benzotriazole esters or PyBOP for coupling under microwave irradiation enhancing the coupling efficiency has been reported. ${ }^{20,21}$ Recently, the solid phase synthesis of $\beta$-peptides containing trans-2-aminocyclohexane carboxylic acid was realized efficiently by microwave irradiation. A comparison of microwave over conventional heating showed that, on treatment with microwave irradiation, the purity of peptide raised to $81 \%$, whereas the conventional method resulted in only $21 \%$ pure compound. ${ }^{22}$ The synthesis of dipeptides containing Z-/Boc- $\alpha$-aminoisobutyric acid (Aib) in solution employing PyBOP/HOBt and HBTU/HOBt was also explored. ${ }^{23}$

\section{Results and Discussion}

In this study, the effect of microwave irradiation on the formation of the peptide bond employing Fmoc-amino acid chlorides has been explored. The microwave reaction was carried out in a LG MS 194A microwave oven producing microwave radiation with a frequency of $2450 \mathrm{MHz}$. The microwave oven was a $1200 \mathrm{~W}$ oven and the reaction was specifically carried out at $60 \%$ of the total power output, which would correspond to an average power of $720 \mathrm{~W}$. For the reactions carried out using a bi-phasic system, the reaction mixture was $\mathrm{CHCl}_{3}-10 \% \mathrm{NaHCO}_{3}$. The synthesis in a homogeneous system in the presence of several hydrogen chloride acceptors was also performed. In the case of the coupling additive TBDMS-OBt, a solution of Fmoc-amino acid chloride in $\mathrm{CH}_{2} \mathrm{Cl}_{2}$ and TBDMS-OBt was used. The zinc, bismuth and indium metal mediated synthesis was carried out using a suspension of metallic powder and Fmoc-amino acid chloride in $\mathrm{CH}_{2} \mathrm{Cl}_{2}$. The completion of the coupling was confirmed by TLC using the solvent systems a) chloroform: methanol: acetic acid (40:2:1) and b) ethyl acetate: $n$-hexane (35:65). A simple work-up of the reaction mixture and recrystallization using a suitable solvent gave the peptide. The duration of the coupling as well as the yield of Fmoc-Phe-Leu-OBzl using various coupling additives in both homogeneous and bi-phasic systems along with the reported data are 
furnished in the Table 1. It has been found that the coupling mediated by zinc dust and TBDMS$\mathrm{OBt}$ is complete within 30 to $45 \mathrm{sec}$. The isolated yield of the pure peptide was $90 \%$. The method was subjected to a racemization test and, as demonstrated by the ${ }^{1} \mathrm{H}$ NMR, and HPLC analysis of the diastereomeric dipeptides, Fmoc-L-Phe- and D-Phe-Ala-OMe, made by employing zinc dust as well as the TBDMS-OBt method, revealed that the method is completely free from racemization. Also, the scale-up of the synthesis of Fmoc-Phe-Leu-OBzl by this method using a 20 mmolar quantity resulted in isolation of $10.8 \mathrm{~g}(92 \%)$ of the pure peptide. Employing a rapid deprotection condition using tris(2-aminoethyl)amine (TAEA), the present method was extended towards the synthesis of Fmoc-Val-Pro-Gly-Val-Gly-OBzl, ${ }^{24}$ a repeating pentapeptide fragment of the protein elastin. Thus, all four of the couplings involved in its synthesis, namely the di, tri, tetra and peptapeptide couplings, have been accomplished under microwave irradiation. The final pure pentapeptide was obtained in $67 \%$ yield (Table 2). The ${ }^{1} \mathrm{H}$ NMR, HPLC (Fig 2) and mass spectrum (Fig 3) of the pentapeptide were found to be satisfactory.

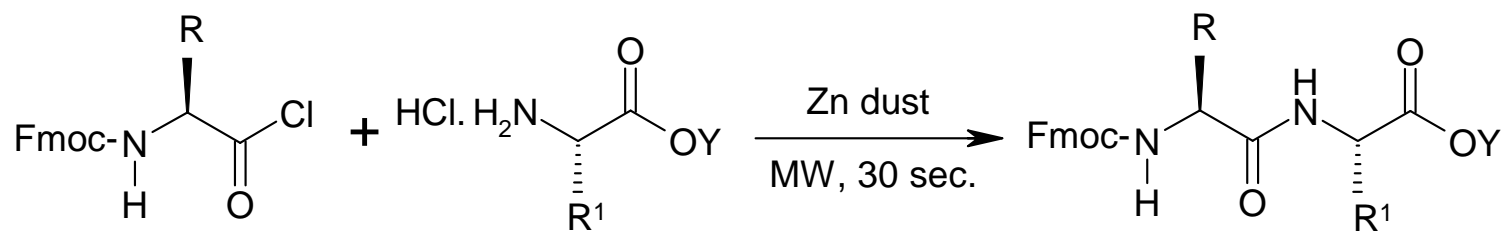

Figure 1. Synthesis of peptides employing zinc dust

Table 1. Synthesis of Fmoc-Phe-Leu-OBzl employing Fmoc-Phe-Cl under various conditions

\begin{tabular}{llcccc}
\hline S1 & Coupling additives & \multicolumn{2}{c}{ Duration of Coupling } & \multicolumn{2}{c}{ Yield (\%) } \\
\cline { 3 - 6 } & & r.t. & $\begin{array}{c}\text { Microwave } \\
\text { conditions }\end{array}$ & r.t. & $\begin{array}{c}\text { Microwave } \\
\text { conditions }\end{array}$ \\
\hline 1. & Zinc (preactivated) & $15 \mathrm{~min}$ & $40 \mathrm{sec}$ & 88 & 92 \\
2. & TBDMS-OBt & $30 \mathrm{~min}$ & $45 \mathrm{sec}$ & 85 & 89 \\
3. & $10 \%$ NaHCO3-CHCl3 & $10 \mathrm{~min}$ & $60 \mathrm{sec}$ & 70 & 60 \\
4. & Bismuth (preactivated) & $2 \mathrm{~h}$ & $2 \mathrm{~min}$ & 65 & 64 \\
5. & Indium & $9 \mathrm{~h}$ & $3 \mathrm{~min}$ & 60 & 40 \\
& & & & & \\
\hline
\end{tabular}


Table 2. List of peptides made by the zinc dust mediated coupling microwave irradiation method

\begin{tabular}{|c|c|c|c|c|c|}
\hline $\begin{array}{l}\text { S1. } \\
\text { No. }\end{array}$ & Peptide ${ }^{*}$ & $\begin{array}{l}\text { M.P. } \\
\left({ }^{\circ} \mathrm{C}\right)\end{array}$ & $\begin{array}{c}\text { Yield } \\
(\%) \\
\end{array}$ & $\begin{array}{l}\text { Time } \\
(\mathrm{sec})\end{array}$ & {$[\alpha]^{25} \mathrm{D}$} \\
\hline 1. & Fmoc-Phg-Phe-OMe & $190-92$ & 93 & 30 & -22.5 (c $0.5, \mathrm{DMF})$ \\
\hline 2. & Fmoc-Phe-Ala-OMe & $183-85$ & 89 & 30 & $-18.0\left(\mathrm{c} 1, \mathrm{CHCl}_{3}\right)$ \\
\hline 3. & Fmoc-D-Phe-Ala-OMe & $186-88$ & 92 & 30 & $+18.3\left(\mathrm{c} 1, \mathrm{CHCl}_{3}\right)$ \\
\hline 4. & Fmoc-Ile-Pro-OMe & $65-67$ & 93 & 30 & $+19.6\left(\mathrm{c} 1, \mathrm{CHCl}_{3}\right)$ \\
\hline 5. & Fmoc-Gly-Phe-OMe & $131-34$ & 92 & 30 & $-16.0(\mathrm{c} 1, \mathrm{MeOH})$ \\
\hline 6. & Fmoc-Met-Val-OMe & $90-93$ & 80 & 30 & $-12.3(\mathrm{c} 1, \mathrm{EtOH})$ \\
\hline 7. & Fmoc-Tyr(Bzl)-Phe-OMe & $171-73$ & 91 & 35 & $+16.3\left(\mathrm{c} 1, \mathrm{CHCl}_{3}\right)$ \\
\hline 8. & Fmoc-Pro-Gly-OBzl & $60-62$ & 79 & 30 & $-27.7\left(\mathrm{c} 1, \mathrm{CHCl}_{3}\right)$ \\
\hline 9. & Fmoc-Leu-Val-OBzl & $125-28$ & 81 & 40 & $-11.8\left(\mathrm{c} 0.5, \mathrm{CHCl}_{3}\right)$ \\
\hline 10. & Fmoc-Phe-Leu-OBzl & $155-58$ & 88 & 35 & $-24.9(\mathrm{c} 1, \mathrm{DMF})$ \\
\hline 11. & Fmoc-Val-Gly-OBzl & $182-84$ & 90 & 30 & $-32.2\left(\mathrm{c} 1, \mathrm{CHCl}_{3}\right)$ \\
\hline 12. & Fmoc-Gly-Val-Gly-OBzl & $176-79$ & 88 & 25 & $-30.4\left(\mathrm{c} 1, \mathrm{CHCl}_{3}\right)$ \\
\hline 13. & Fmoc-Pro-Gly-Val-Gly-OBzl & $158-60$ & 78 & 30 & $-24.2\left(\mathrm{c} 1, \mathrm{CHCl}_{3}\right)$ \\
\hline 14. & Fmoc-Val-Pro-Gly-Val-Gly-OBzl & $93-95$ & 67 & 30 & $-29.2\left(\mathrm{c} 1, \mathrm{CHCl}_{3}\right)$ \\
\hline
\end{tabular}

${ }^{* 1} \mathrm{H}$ NMR of all the peptides obtained was satisfactory

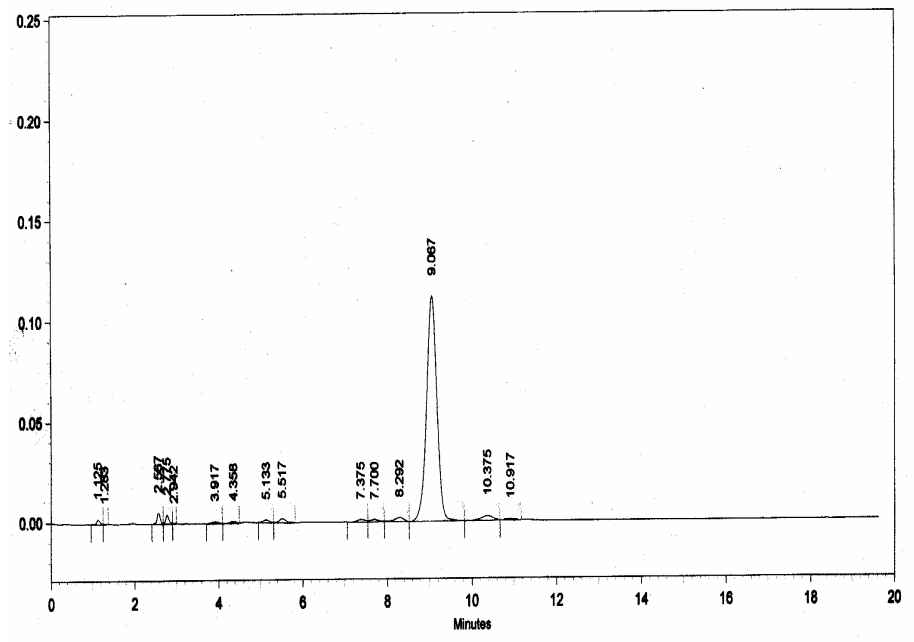

Figure 2. The HPLC of Fmoc-Val-Pro-Gly-Val-Gly-OBzl detection at $210 \mathrm{~nm}$; eluents: acetonitrile $(60 \%)$ and water $(40 \%)$; flow rate of $1.0 \mathrm{~mL} / \mathrm{min}$; $\mathrm{R}_{t}$ value : $9.06 \mathrm{~min}$., purity $>90 \%$ 


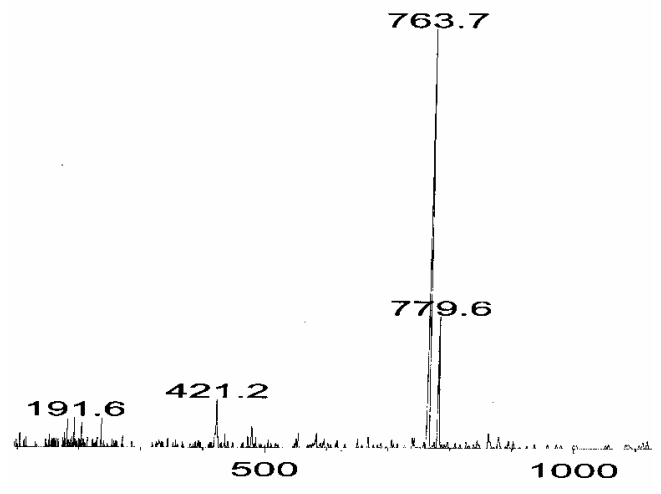

Figure 3. ES MS; $[\mathrm{M}+\mathrm{Na}]^{+}: 763.7,[\mathrm{M}+\mathrm{K}]^{+}: 779.6$

Encouraged by these results, we have extended the similar reaction conditions for the incorporation of $\alpha, \alpha$-dialkylamino acids. The formation of a peptide bond between $\alpha, \alpha$ dialkylamino acids, both acyclic and cyclic, under normal conditions employing even efficient coupling agents like PyBOP, HBTU etc., needs several hours with poor yields. Even under microwave irradiation, the synthesis of Boc-/Z-Aib-Aib-OMe using HBTU/HOBt required 30 min. It is now found that the coupling of Fmoc-Aib-Cl to Aib-OBzl hydrochloride, mediated by activated zinc dust under microwave irradiation, has been found to be complete within $90 \mathrm{sec}$. with $84 \%$ yield. Similar results have been obtained in the coupling of several other peptides possessing cyclic as well as acyclic $\alpha, \alpha$-dialkylamino acids, and these are summarized in Table 3.

Table 3. List of dipeptides containing dialkyl amino acids synthesized employing Fmoc- $\alpha, \alpha-$ dialkyl amino acid chloride and zinc dust under microwave irradiation

\begin{tabular}{lllll}
\hline $\begin{array}{l}\text { Sl. } \\
\text { No. }\end{array}$ & Peptide $^{*}$ & $\begin{array}{l}\text { M.P. } \\
\left({ }^{\circ} \mathrm{C}\right)\end{array}$ & $\begin{array}{l}\text { Yield } \\
(\%)\end{array}$ & $\begin{array}{l}\text { Time } \\
(\mathrm{min})\end{array}$ \\
\hline 1. & Fmoc-Aib-Aib-OBzl & $131-32$ & 84 & 1.5 \\
2. & Fmoc-Ac $_{6} \mathrm{c}-\mathrm{Ac}_{6} \mathrm{c}-\mathrm{OMe}$ & $164-65$ & 89 & 2.0 \\
3. & Fmoc-Ac $_{6} \mathrm{c}-\mathrm{Ac}_{6} \mathrm{c}-\mathrm{Ac}_{6} \mathrm{c}-\mathrm{OMe}$ & $185-88$ & 79 & 2.0 \\
4. & Fmoc-Ac ${ }_{7} \mathrm{c}-\mathrm{Ac}_{7} \mathrm{c}-\mathrm{OMe}$ & $207-09$ & 80 & 2.5 \\
5. & Fmoc-Deg-Deg-OMe & $122-25$ & 81 & 2.5 \\
6. & Fmoc-Dpg-Dpg-OMe & $128-29$ & 89 & 2.0 \\
7. & Fmoc-Dpg-Dpg-Dpg-OMe & $151-22$ & 90 & 2.5 \\
8. & Fmoc-Dphg-Dphg-OMe & $198-200$ & 88 & 2.5 \\
9. & Fmoc-Dphg-Aib-OBzl & $170-73$ & 90 & 2.5 \\
\hline
\end{tabular}

- ${ }^{1} \mathrm{H}$ NMR and mass spectra of all the products were satisfactory

- Aib, 2-amino isobutyric acid; Dpg, diisopropyl glycine; Dphg, diphenyl glycine; Ac 6 c, 1amino cyclohexane carboxylic acic; $\mathrm{Ac}_{7} \mathrm{c}, 1$-amino cycloheptane carboxylic acid. 


\section{Conclusions}

The synthesis of peptides employing Fmoc-amino acid chlorides using a mild microwave irradiation technique in the solution phase has been described. The method achieves the purpose of high speed and efficient coupling of peptides, including those of highly hindered $\alpha, \alpha-$ dialkylamino acids. Thus, a facile method to produce peptides in high yield makes this yet another useful and attractive procedure employing the microwave technique in organic synthesis.

\section{Experimental Section}

General Procedures. All the solvents were freshly distilled prior to use. Melting points were determined by the capillary method and are uncorrected. TLC analysis was carried out on precoated silica gel- $\mathrm{GF}_{254}$ plates purchased from Merck containing $13 \% \mathrm{CaSO}_{4}$ as binding agent, using solvent systems a) chloroform : methanol : acetic acid (40:2:1) and b) ethyl acetate : $n$ hexane (35:65). IR spectra were recorded on a Nicolet model impact 400D FT-IR spectrometer. The HPLC analysis was carried on Shimadzu CLASS-VP V6.1 analytical HPLC using a Merck $\mathrm{RP}-18,250 \times 4.0 \mathrm{~mm}$ column, with detection at $215 \mathrm{~nm}$. Optical rotations were determined using automatic AA-10 polarimeter (Optical Activity, U. K.). Elemental analyses were carried out on a Perkin-Elmer MODEL 240 analyzer. ${ }^{1} \mathrm{H}$ NMR spectra were recorded using a Bruker AMX 400 $\mathrm{MHz}$ spectrometer. Mass spectra were obtained using a Kratos PCKompact SEQ V1.2.2 spectrometer.

General procedure for the synthesis of peptides mediated by zinc dust under MW irradiation. A beaker containing a mixture of Fmoc-amino acid chloride $(1 \mathrm{mmol})$, zinc dust $(140 \mathrm{mg}, 2 \mathrm{mmol})$ and amino acid ester hydrochloride $(1.05 \mathrm{mmol})$ in DCM $(10 \mathrm{~mL})$ was irradiated with microwave (at P-60) for 30-40 sec. (1.5-2.5 min. in case of dialkyl amino acids). After completion of the reaction, the residue was diluted with $\mathrm{CHCl}_{3}(25 \mathrm{~mL})$, washed with $5 \%$ $\mathrm{HCl}\left(5 \mathrm{~mL} \mathrm{X} \mathrm{2),} 10 \% \mathrm{NaHCO}_{3}(5 \mathrm{~mL} \mathrm{X} \mathrm{2)}\right.$ ), and water ( $5 \mathrm{~mL} \mathrm{X} \mathrm{2).} \mathrm{The} \mathrm{organic} \mathrm{layer} \mathrm{was} \mathrm{dried}$ over anhyd. $\mathrm{Na}_{2} \mathrm{SO}_{4}$ and evaporated under reduced pressure. Recrystallization of the resulting residue with DCM: $n$-hexane (1:3) gave the pure peptide.

General procedure for the deprotection of Fmoc-peptides using tris (2-aminoethyl)amine (TAEA). TAEA $(5 \mathrm{~mL})$ was added to a solution of Fmoc- protected peptide $(1 \mathrm{mmol})$ in DCM $(5 \mathrm{~mL})$ and stirred for $20 \mathrm{~min}$. After completion of the reaction, the solution was further diluted with DCM $(30 \mathrm{~mL})$, washed with phosphate buffer $(5 \mathrm{~mL} \mathrm{X} \mathrm{3)}$ and water $(5 \mathrm{~mL} \mathrm{X} \mathrm{3)}$. The organic layer was dried over $\mathrm{Na}_{2} \mathrm{SO}_{4}$ and taken for the coupling of next amino acid.

Racemization studies Following the general procedure for the synthesis of peptides mediated by zinc dust, a mixture of Fmoc-Phe-Cl/Fmoc-D-Phe-Cl (400 mg, $1 \mathrm{mmol}$ ), zinc dust (140 mg, 2 $\mathrm{mmol})$ and HCl.H-Ala-OMe $(145 \mathrm{mg}, 1.05 \mathrm{mmol})$ in DCM $(10 \mathrm{~mL})$ resulted in Fmoc-Phe-AlaOMe (420 mg, 89\%) and Fmoc-D-Phe-Ala-OMe (440 mg, 92\%) of the pure peptide. The HPLC analysis was carried on Shimadzu CLASS-VP V6.1 analytical HPLC using a Merck RP-18, 250 
x $4.0 \mathrm{~mm}$ column, with detection at $215 \mathrm{~nm}$. The eluent was acetonitrile (50\%) and water (50\%) with the flow rate of $1.0 \mathrm{~mL} / \mathrm{min}$; $\mathrm{R}_{t}$ value for $\mathrm{L}$, $\mathrm{L}$ diastereomer : $16.62 \mathrm{~min}$.; $\mathrm{R}_{t}$ value for $\mathrm{D}, \mathrm{L}$ diastereomer : $17.56 \mathrm{~min}$.

Fmoc-Phg-Phe-OMe. White solid (93\%), ${ }^{1} \mathrm{H}$ NMR $\delta\left(400 \mathrm{MHz}, \mathrm{CDCl}_{3}\right): 2.96(\mathrm{~d}, \mathrm{~J}=5.7 \mathrm{~Hz}$, 2H), $3.67(\mathrm{~s}, 3 \mathrm{H}), 4.05(\mathrm{~m}, 1 \mathrm{H}), 4.21(\mathrm{t}, \mathrm{J}=6.6 \mathrm{~Hz}, 1 \mathrm{H}), 4.39(\mathrm{~d}, \mathrm{~J}=6.7 \mathrm{~Hz}, 2 \mathrm{H}), 4.72(\mathrm{~m}, 1 \mathrm{H}), 5.3$ (br s, 1H), 6.26 (br s, 1H), 6.97-7.69 (m, 18H). Anal. Calcd. for $\mathrm{C}_{33} \mathrm{H}_{30} \mathrm{~N}_{2} \mathrm{O}_{5}$ (534.62): C, 74.14; H, 5.66; N, 5.24. Found: C, 74.02; H, 5.57; N, 5.14. ES MS: $m / z=557.4[\mathrm{M}+\mathrm{Na}]^{+}$.

Fmoc-Phe-Ala-OMe. White solid (89\%), ${ }^{1} \mathrm{H}$ NMR $\delta\left(400 \mathrm{MHz}, \mathrm{CDCl}_{3}\right): 1.26(\mathrm{~d}, \mathrm{~J}=7.1,3 \mathrm{H})$, $2.98(\mathrm{~d}, \mathrm{~J}=7.0 \mathrm{~Hz}, 2 \mathrm{H}), 3.63(\mathrm{~s}, 3 \mathrm{H}), 4.12(\mathrm{t}, \mathrm{J}=6.8 \mathrm{~Hz}, 1 \mathrm{H}), 4.25-4.45(\mathrm{~m}, 4 \mathrm{H}), 5.31$ (br s, 1H), 6.26 (br s, 1H), 7.10- 7.70 (ArH, 13H). Anal. Calcd. for $\mathrm{C}_{28} \mathrm{H}_{28} \mathrm{~N}_{2} \mathrm{O}_{5}$ (472.55): C, 71.17; H, 5.97; N, 5.93. Found: C, 71.09; H, 5.87; N, 5.90. ES MS: $\mathrm{m} / \mathrm{z}=495.5[\mathrm{M}+\mathrm{Na}]^{+}$.

Fmoc-D-Phe-Ala-OMe. White solid (92\%), ${ }^{1} \mathrm{H}$ NMR $\delta\left(400 \mathrm{MHz}, \mathrm{CDCl}_{3}\right): 1.30(\mathrm{~d}, \mathrm{~J}=7.1,3 \mathrm{H})$, $3.06(\mathrm{~d}, \mathrm{~J}=6.9 \mathrm{~Hz}, 2 \mathrm{H}), 3.71(\mathrm{~s}, 3 \mathrm{H}), 4.15(\mathrm{t}, \mathrm{J}=6.8 \mathrm{~Hz}, 1 \mathrm{H}), 4.25-4.45(\mathrm{~m}, 4 \mathrm{H}), 5.30$ (br s, 1H), 6.26 (br s, 1H), 7.13- 7.70 (ArH, 13H). Anal. Calcd. for $\mathrm{C}_{28} \mathrm{H}_{28} \mathrm{~N}_{2} \mathrm{O}_{5}$ (472.55): C, 71.17; $\mathrm{H}$, 5.97; N, 5.93. Found: C, 71.15; H, 5.91; N, 5.92. ES MS: $\mathrm{m} / \mathrm{z}=495.7[\mathrm{M}+\mathrm{Na}]^{+}$.

Fmoc-Ile-Pro-OMe. Colourless oil solidifies on standing (93\%), ${ }^{1} \mathrm{H}$ NMR $\delta(400 \mathrm{MHz}$, $\left.\mathrm{CDCl}_{3}\right): 0.81(\mathrm{~m}, 6 \mathrm{H}), 1.13(\mathrm{~m}, 1 \mathrm{H}), 1.22-2.05(\mathrm{~m}, 6 \mathrm{H}), 3.60(\mathrm{~m}, 2 \mathrm{H}), 3.68(\mathrm{~s}, 3 \mathrm{H}), 4.03(\mathrm{~m}, 2 \mathrm{H})$, $4.21(\mathrm{t}, \mathrm{J}=6.6 \mathrm{~Hz}, 1 \mathrm{H}), 4.44(\mathrm{~m}, 2 \mathrm{H}), 5.91(\mathrm{br} \mathrm{s}, 1 \mathrm{H}), 7.25-7.76(\mathrm{~m}, 8 \mathrm{H})$. Anal. Calcd. for $\mathrm{C}_{27} \mathrm{H}_{32}$ $\mathrm{N}_{2} \mathrm{O}_{5}$ (464.57): C, 69.81; H, 6.94; N, 6.03. Found: C, 69.78; H, 6.91; N, 5.97. ES MS: m/z $=487.8[\mathrm{M}+\mathrm{Na}]^{+}$.

Fmoc-Gly-Phe-OMe. White solid (92\%), ${ }^{1} \mathrm{H}$ NMR $\delta\left(400 \mathrm{MHz}, \mathrm{CDCl}_{3}\right): 2.87$ (d, 2H, J=6.2 $\mathrm{Hz}), 3.69$ (s, 3H), $4.03(\mathrm{~m}, 3 \mathrm{H}), 4.18$ (t, J=6.6 Hz, 1H), $4.40(\mathrm{~m}, 2 \mathrm{H}), 5.96$ (br s, 1H), 6.2 (br s, 1H), 7.1-7.76 (m, 13H). Anal. Calcd. for $\mathrm{C}_{27} \mathrm{H}_{26} \mathrm{~N}_{2} \mathrm{O}_{5}$ (458.52): C, 70.73; H, 5.72; N, 6.11. Found: C, 70.69.09; H, 5.67; N, 6.08. ES MS: m/z=481.1 [M+ Na $]^{+}$.

Fmoc-Met-Val-OMe. Yellowish residue slowly crystallizes to give solid $(80 \%),{ }^{1} \mathrm{H}$ NMR $\delta$ $\left(400 \mathrm{MHz}, \mathrm{CDCl}_{3}\right): 0.93(2 \mathrm{~d}, \mathrm{~J}=6 \mathrm{~Hz}, 6 \mathrm{H}), 1.81-2.55(\mathrm{~m}, 5 \mathrm{H}), 2.78(\mathrm{~s}, 3 \mathrm{H}), 3.62(\mathrm{~s}, 3 \mathrm{H}), 3.75$ $(\mathrm{m}, 1 \mathrm{H}), 4.05(\mathrm{~m}, 1 \mathrm{H}), 4.21(\mathrm{t}, \mathrm{J}=6.6,1 \mathrm{H}), 4.50(\mathrm{~d}, \mathrm{~J}=6 \mathrm{~Hz}, 2 \mathrm{H}), 5.05(\mathrm{~d}, \mathrm{~J}=8.0 \mathrm{~Hz}, 1 \mathrm{H}),$,6.10 ( br, s, $1 \mathrm{H})$, 7.26-7.7 (m, 8H). Anal. Calcd. for $\mathrm{C}_{26} \mathrm{H}_{32} \mathrm{~N}_{2} \mathrm{O}_{5} \mathrm{~S}_{1}$ (484.62): C, 64.44; $\mathrm{H}, 6.66$; $\mathrm{N}$, 5.78. Found: C, 64.33; H, 6.61; N, 5.75. ES MS: $\mathrm{m} / \mathrm{z}=507.3[\mathrm{M}+\mathrm{Na}]^{+}$.

Fmoc-Tyr(Bzl)-Phe-OMe. White solid (91\%), ${ }^{1} \mathrm{H}$ NMR $\delta\left(400 \mathrm{MHz}, \mathrm{CDCl}_{3}\right): 2.93$ (m, $\left.2 \mathrm{H}\right)$, $3.08(\mathrm{~m}, 2 \mathrm{H}), 3.67(\mathrm{~s}, 3 \mathrm{H}), 4.00-4.09(\mathrm{~m}, 2 \mathrm{H}), 4.16(\mathrm{t}, \mathrm{J}=6.6 \mathrm{~Hz}, 1 \mathrm{H}), 4.44(\mathrm{~d}, \mathrm{~J}=6.7 \mathrm{~Hz}, 2 \mathrm{H})$, $5.16(\mathrm{~m}, 2 \mathrm{H}), 5.71(\mathrm{~d}, \mathrm{~J}=8.6 \mathrm{~Hz}, 1 \mathrm{H}), 5.82(\mathrm{~m}, \mathrm{~J}=8.8 \mathrm{~Hz}, 1 \mathrm{H}), 7.26-7.8(\mathrm{~m}, 22 \mathrm{H})$. Anal. Calcd. for $\mathrm{C}_{41} \mathrm{H}_{38} \mathrm{~N}_{2} \mathrm{O}_{6}$ (654.77): C, 75.21; H, 5.85; N, 4.28. Found: C, 75.22; H, 5.88; N, 4.38. ES MS: $\mathrm{m} / \mathrm{z}=677.9[\mathrm{M}+\mathrm{Na}]^{+}$.

Fmoc-Pro-Gly-OMe. Viscous liquid solidifies on standing to give solid (79\%), ${ }^{1} \mathrm{H}$ NMR $\delta(400$ $\left.\mathrm{MHz}, \mathrm{CDCl}_{3}\right)$ : 1.22-2.05 (m, 4H), $3.33(\mathrm{~d}, 2 \mathrm{H}), 3.62(\mathrm{~s}, 3 \mathrm{H}), 3.90(\mathrm{~m}, 2 \mathrm{H}), 4.03(\mathrm{~m}, 1 \mathrm{H}), 4.19(\mathrm{t}$, $\mathrm{J}=6.6 \mathrm{~Hz}, 1 \mathrm{H}), 4.38(\mathrm{~m}, 2 \mathrm{H}), 5.18$ (br s, $1 \mathrm{H}), 7.26-7.8(\mathrm{~m}, 8 \mathrm{H})$. Anal. Calcd. for $\mathrm{C}_{23} \mathrm{H}_{24} \mathrm{~N}_{2} \mathrm{O}_{5}$ (408.45): C, 67.3; H, 5.92; N, 6.85. Found: C, 69.11; H, 5.88; N, 6.75. ES MS: m/z =431.9 [M + $\mathrm{Na}]^{+}$. 
Fmoc-Leu-Val-OBzl. White solid (81\%), ${ }^{1} \mathrm{H} \mathrm{NMR} \delta\left(400 \mathrm{MHz}, \mathrm{CDCl}_{3}\right): 0.83-1.11(\mathrm{~m}, 12 \mathrm{H})$, $1.33(\mathrm{~m}, 1 \mathrm{H}), 1.63(\mathrm{~m}, 2 \mathrm{H}), 2.1(\mathrm{~m}, 1 \mathrm{H}), 4.09(\mathrm{~m}, 1 \mathrm{H}), 4.19(\mathrm{t}, \mathrm{J}=6.5 \mathrm{~Hz}, 1 \mathrm{H}), 4.34(\mathrm{~m}, 1 \mathrm{H})$, $4.45(\mathrm{~d}, \mathrm{~J}=6.6 \mathrm{~Hz}, 2 \mathrm{H}), 5.14(\mathrm{~m}, 2 \mathrm{H}), 5.55(\mathrm{~d}, \mathrm{~J}=7.2 \mathrm{~Hz}, 1 \mathrm{H}), 6.2(\mathrm{~d}, \mathrm{~J}=8.2 \mathrm{~Hz}, 1 \mathrm{H}), 7.26-7.8$ (m, 13H). Anal. Calcd. for $\mathrm{C}_{33} \mathrm{H}_{38} \mathrm{~N}_{2} \mathrm{O}_{5}$ (542.68): C, 73.04; H, 7.06; N, 5.17. Found: C, 72.95; H, 6.99; N, 5.06. ES MS: $\mathrm{m} / \mathrm{z}=565.6[\mathrm{M}+\mathrm{Na}]^{+}$.

Fmoc-Phe-Leu-OBzl. White solid (88\%), ${ }^{1} \mathrm{H}$ NMR $\delta\left(400 \mathrm{MHz}, \mathrm{CDCl}_{3}\right): 0.91$ (d, J=5.2 Hz, $6 \mathrm{H}), 1.33(\mathrm{~m}, 1 \mathrm{H}), 1.63(\mathrm{~m}, 2 \mathrm{H}), 2.87(\mathrm{~d}, \mathrm{~J}=6.2 \mathrm{~Hz}, 2 \mathrm{H}), 3.96(\mathrm{~m}, 1 \mathrm{H}), 4.05(\mathrm{~m}, 1 \mathrm{H}), 4.20(\mathrm{t}, \mathrm{J}=$ $6.6 \mathrm{~Hz}, 1 \mathrm{H}), 4.54(\mathrm{~m}, 2 \mathrm{H}), 5.13(\mathrm{~m}, 2 \mathrm{H}), 5.5$ (br s, 1H), 6.21 (br s, 1H), 7.26-7.8 (m, 18H). Anal. Calcd. for $\mathrm{C}_{37} \mathrm{H}_{38} \mathrm{~N}_{2} \mathrm{O}_{5}$ (590.73): C, 75.23; H, 6.48; N, 4.74. Found: C, 75.19; H, 6.42; N, 4.70. ES MS: $\mathrm{m} / \mathrm{z}=613.7[\mathrm{M}+\mathrm{Na}]^{+}$.

\section{Synthesis of Fmoc-Val-Pro-Gly-Val-Gly-OBzl}

Fmoc-Val-Gly-OBzl. White solid (90\%), ${ }^{1} \mathrm{H}$ NMR $\delta\left(400 \mathrm{MHz}, \mathrm{CDCl}_{3}\right): \delta$ 0.9-1.01 (2d, J=6.1 $\mathrm{Hz}, 6 \mathrm{H}), 2.01(\mathrm{~m}, 1 \mathrm{H}), 3.67(\mathrm{~d}, 2 \mathrm{H}), 3.93(\mathrm{~m}, 1 \mathrm{H}), 4.18(\mathrm{t}, \mathrm{J}=6.5 \mathrm{~Hz}, 1 \mathrm{H}), 4.48$ (d, J=6.1 Hz, $2 \mathrm{H}), 5.05(\mathrm{~m}, 2 \mathrm{H}), 5.21$ (br s, $1 \mathrm{H}), 5.66($ br s, $1 \mathrm{H}), 7.27-7.68(\mathrm{~m}, 13 \mathrm{H})$. Anal. Calcd. for $\mathrm{C}_{29} \mathrm{H}_{30}$ $\mathrm{N}_{2} \mathrm{O}_{5}$ (486.57): C, 71.59; H, 6.21; N, 5.76. Found: C, 71.55; H, 6.19; N, 5.73. ES MS: m/z $=509.3[\mathrm{M}+\mathrm{Na}]^{+}$.

Fmoc-Gly-Val-Gly-OBzl. White solid (88\%), ${ }^{1} \mathrm{H}$ NMR $\delta\left(400 \mathrm{MHz}, \mathrm{CDCl}_{3}\right): \delta 0.9-1.01(2 \mathrm{~d}, \mathrm{~J}=$ $6.6 \mathrm{~Hz}, 6 \mathrm{H}), 2.11(\mathrm{~m}, 1 \mathrm{H}), 3.67(\mathrm{~m}, 4 \mathrm{H}), 3.93(\mathrm{~m}, 1 \mathrm{H}), 4.18(\mathrm{t}, \mathrm{J}=55 \mathrm{~Hz}, 1 \mathrm{H}), 4.45(\mathrm{~m}, 2 \mathrm{H}), 5.07$ $(\mathrm{d}, 2 \mathrm{H}), 5.21(\mathrm{~m}, 2 \mathrm{H}), 6.2(\mathrm{~d}, 1 \mathrm{H}), 7.21-7.75(\mathrm{~m}, 13 \mathrm{H})$. Anal. Calcd. for $\mathrm{C}_{31} \mathrm{H}_{33} \mathrm{~N}_{3} \mathrm{O}_{6}(543.63)$ : C, 68.49; H, 6.12; N, 7.73. Found: C, 68.55; H, 6.19; N, 7.79. ES MS: m/z =566.2 [M + Na $]^{+}$.

Fmoc-Pro-Gly-Val-Gly-OBzl. White solid (78\%), ${ }^{1} \mathrm{H}$ NMR $\delta\left(400 \mathrm{MHz}, \mathrm{CDCl}_{3}\right): \delta$ 0.9-1.01 $(2 \mathrm{~d}, \mathrm{~J}=6.7 \mathrm{~Hz}, 6 \mathrm{H}), 1.73-2.24(\mathrm{~m}, 5 \mathrm{H}), 3.67(\mathrm{~m}, 2 \mathrm{H}), 3.93-4.12(\mathrm{~m}, 6 \mathrm{H}), 4.19$ (t, J= 5.6 Hz, 1H), $4.45(\mathrm{~m}, 2 \mathrm{H}), 5.07(\mathrm{~m}, 2 \mathrm{H}), 5.11(\mathrm{~m}, 1 \mathrm{H}), 5.19(\mathrm{~m}, 1 \mathrm{H}), 6.00(\mathrm{~d}, \mathrm{~J}=9.0 \mathrm{~Hz}, 1 \mathrm{H}), 7.21-7.75(\mathrm{~m}$, 13H). Anal. Calcd. for $\mathrm{C}_{36} \mathrm{H}_{40} \mathrm{~N}_{4} \mathrm{O}_{7}$ (640.74): C, 67.48; H, 6.29; N, 8.74. Found: C, 67.20; H, 6.29; N, 8.73. ES MS: $\mathrm{m} / \mathrm{z}=663.7[\mathrm{M}+\mathrm{Na}]^{+}$.

Fmoc-Val-Pro-Gly-Val-Gly-OBzl. Sowly crystallizes to result in white solid (67\%), ${ }^{1} \mathrm{H}$ NMR $\delta$ $\left(400 \mathrm{MHz}, \mathrm{CDCl}_{3}\right): \delta$ 0.83-1.01 (m, 12H), 1.42-2.14 (m, 6H), 3.33( m, 2H), $3.63(\mathrm{~m}, 2 \mathrm{H}), 3.93$ $(\mathrm{m}, 2 \mathrm{H}), 4.12(\mathrm{~m}, 1 \mathrm{H}), 4.21(\mathrm{t}, \mathrm{J}=8.7 \mathrm{~Hz}, 1 \mathrm{H}), 4.34(\mathrm{~m}, 2 \mathrm{H}), 4.45(\mathrm{~d}, \mathrm{~J}=5.8 \mathrm{~Hz}, 2 \mathrm{H}), 5.07(\mathrm{~m}$, 2H), 5.11-5.19 (m, 2H), 5.37 (br s, 1H), $6.00(\mathrm{~d}, \mathrm{~J}=9.0 \mathrm{~Hz}, 1 \mathrm{H}), 7.21-7.75$ (m, 13H). Anal. Calcd. for $\mathrm{C}_{41} \mathrm{H}_{49} \mathrm{~N}_{5} \mathrm{O}_{8}$ (739.98): C, 66.56; H, 6.68; N, 9.47. Found: C, 66.47; H, 6.57; N, 9.40. ES MS: $\mathrm{m} / \mathrm{z}=763.7[\mathrm{M}+\mathrm{Na}]^{+}$.

Fmoc-Aib-Aib-OBzl. White solid (84\%), ${ }^{1} \mathrm{H}$ NMR $\delta\left(400 \mathrm{MHz}, \mathrm{CDCl}_{3}\right): 1.53(\mathrm{~s}, 6 \mathrm{H}), 1.58$ (s, $6 \mathrm{H}), 4.18(\mathrm{t}, \mathrm{J}=6.4 \mathrm{~Hz}, 1 \mathrm{H}), 4.44(\mathrm{~d}, \mathrm{~J}=6.3 \mathrm{~Hz}, 2 \mathrm{H}), 5.1(\mathrm{~s}, 2 \mathrm{H}), 5.34$ (d, J= $7.7 \mathrm{~Hz}, 1 \mathrm{H}), 6.16$ (d, $8.8 \mathrm{~Hz}, 1 \mathrm{H}), 7.26-7.8(\mathrm{~m}, 13 \mathrm{H})$. Anal. Calcd. for $\mathrm{C}_{30} \mathrm{H}_{32} \mathrm{~N}_{2} \mathrm{O}_{5}(500.60): \mathrm{C}, 71.98 ; \mathrm{H}, 6.44 ; \mathrm{N}$, 5.60. Found: C, 71.88; H, 6.37; N, 5.56. ES MS: $\mathrm{m} / \mathrm{z}=523.9[\mathrm{M}+\mathrm{Na}]^{+}$.

Fmoc-Ac $\mathbf{c}_{\mathbf{6}} \mathbf{c}-\mathbf{A c}_{\mathbf{6}} \mathbf{c}-\mathbf{O M e}$. White solid (89\%), ${ }^{1} \mathrm{H} \mathrm{NMR} \delta\left(300 \mathrm{MHz}, \mathrm{CDCl}_{3}\right): 0.93-2.17$ (m, 20H), $3.67(\mathrm{~s}, 3 \mathrm{H}), 4.22(\mathrm{t}, \mathrm{J}=6.8 \mathrm{~Hz}, 1 \mathrm{H}), 4.40$ (d, J=6.9 Hz, 2H), 5.8 (br s, 1H), 6.3 (br s, 1H), 7.247.1 (m, 8H). Anal. Calcd. for $\mathrm{C}_{29} \mathrm{H}_{36} \mathrm{~N}_{2} \mathrm{O}_{5}$ (492.61): C, 70.70; H, 7.36; N, 5.68. Found: C, 70.75; H, 7.39; N, 5.73. ES MS: $\mathrm{m} / \mathrm{z}=516.1[\mathrm{M}+\mathrm{Na}]^{+}$. 
Fmoc-Ac $\mathbf{F}_{\mathbf{6}} \mathbf{c}-\mathbf{A c}_{\mathbf{6}} \mathbf{c}-\mathbf{A c}_{\mathbf{6}} \mathbf{c}-\mathbf{O M e}$. White solid (79\%), ${ }^{1} \mathrm{H}$ NMR $\delta\left(300 \mathrm{MHz}, \mathrm{CDCl}_{3}\right)$ : 0.93-2.27 (m, $30 \mathrm{H}), 3.7$ (s, 3H), 4.19 (t, J=6.6 Hz, 1H), 4.3 (d, J=6.5 Hz, 2H), 5.2 (br s, 1H), 5.6 (br s, 1H), 6.3 (br s, 1H), 7.23-7.82 (m, 8H). Anal. Calcd. for $\mathrm{C}_{36} \mathrm{H}_{47} \mathrm{~N}_{3} \mathrm{O}_{6}(617.78)$ : C, 69.99; H, 7.66; N, 6.8. Found: C, 70.08; H, 7.72; N, 6.71. ES MS: m/z=641.5 $[\mathrm{M}+\mathrm{Na}]^{+}$.

Fmoc-Ac $\mathbf{F c}_{7} \mathbf{c}-\mathbf{A c}_{7} \mathbf{c}-\mathbf{O M e}$. White solid (80\%), ${ }^{1} \mathrm{H} \mathrm{NMR} \delta\left(300 \mathrm{MHz}, \mathrm{CDCl}_{3}\right):$ 0.76-2.31 (m, 24H), $3.7(\mathrm{~s}, 3 \mathrm{H}), 4.1(\mathrm{t}, \mathrm{J}=6.5 \mathrm{~Hz}, 1 \mathrm{H}), 4.3(\mathrm{~m}, 2 \mathrm{H}), 5.3$ (br s, 1H), 6.1 (br s, 1H), 7.25-7.77 (m, 8H). Anal. Calcd. for $\mathrm{C}_{31} \mathrm{H}_{40} \mathrm{~N}_{2} \mathrm{O}_{5}$ (520.63): C, 71.51; H, 7.73; N, 5.38. Found: C, 71.038; H, 7.65; $\mathrm{N}$, 5.25. ES MS: $\mathrm{m} / \mathrm{z}=543.9[\mathrm{M}+\mathrm{Na}]^{+}$.

Fmoc-Deg-Deg-OMe. White solid (81\%), ${ }^{1} \mathrm{H}$ NMR $\delta\left(400 \mathrm{MHz}, \mathrm{CDCl}_{3}\right)$ : 0.76- 0.87(m, 12H), $1.79(\mathrm{~m}, 8 \mathrm{H}), 3.67(\mathrm{~s}, 3 \mathrm{H}), 4.18(\mathrm{t}, \mathrm{J}=6.6 \mathrm{~Hz}, 1 \mathrm{H}), 4.4(\mathrm{~d}, \mathrm{~J}=5.9 \mathrm{~Hz}, 2 \mathrm{H}), 5.26$ (br s, $1 \mathrm{H}), 6.4$ (br s, 1H), 7.27- $7.80(\mathrm{~m}, 8 \mathrm{H})$. Anal. Calcd. for $\mathrm{C}_{28} \mathrm{H}_{36} \mathrm{~N}_{2} \mathrm{O}_{5}$ (481.16): C, 69.89; H, 7.65; N, 5.82. Found: C, 70.01; H, 7.72; N, 5.71. ES MS: m/z=504.7 [M+ Na $]^{+}$.

Fmoc-Dpg-Dpg-OMe. White solid (89\%), ${ }^{1} \mathrm{H}$ NMR $\delta\left(400 \mathrm{MHz}, \mathrm{CDCl}_{3}\right): 0.82-0.94(\mathrm{~m}, 12 \mathrm{H})$, 0.99-1.13 (m, 8H), 1.18-1.32 (m, 8H), $3.68(\mathrm{~s}, 3 \mathrm{H}), 4.18(\mathrm{t}, \mathrm{J}=6.4 \mathrm{~Hz}, 1 \mathrm{H}), 4.43$ (d, J=6.6 Hz, 2H), 4.5 (br s, $1 \mathrm{H}), 5.6$ (br s, $1 \mathrm{H}), 7.28-7.8(\mathrm{~m}, 8 \mathrm{H})$. Anal. Calcd. for $\mathrm{C}_{32} \mathrm{H}_{44} \mathrm{~N}_{2} \mathrm{O}_{5}(536.38)$ : C, 71.59; H, 8.26; N, 5.22. Found: C, 79.66; H, 8.14; N, 5.14. ES MS: m/z =559.9 $[\mathrm{M}+\mathrm{Na}]^{+}$.

Fmoc-Dpg-Dpg-Dpg-OMe. White solid (90\%), ${ }^{1} \mathrm{H}$ NMR $\delta\left(400 \mathrm{MHz}, \mathrm{CDCl}_{3}\right)$ : 0.82-0.94 (m, $18 \mathrm{H}), 0.99-1.13(\mathrm{~m}, 12 \mathrm{H}), 1.18-1.32(\mathrm{~m}, 12 \mathrm{H}), 3.68(\mathrm{~s}, 3 \mathrm{H}), 4.18(\mathrm{t}, \mathrm{J}=6.4 \mathrm{~Hz}, 1 \mathrm{H}), 4.43(\mathrm{~d}, \mathrm{~J}=$ $6.6 \mathrm{~Hz}, 2 \mathrm{H}), 5.5(\mathrm{br} \mathrm{s}, 1 \mathrm{H}), 5.6(\mathrm{br} \mathrm{s}, 1 \mathrm{H}), 6.1(\mathrm{~s}, 1 \mathrm{H}), 7.28-7.8(\mathrm{~m}, 8 \mathrm{H})$. Anal. Calcd. for $\mathrm{C}_{40} \mathrm{H}_{59}$ $\mathrm{N}_{3} \mathrm{O}_{6}$ (677.52): C, 70.85; H, 8.77; N, 6.20. Found: C, 70.70; H, 8.72; N, 6.15. ES MS: m/z $=700.9[\mathrm{M}+\mathrm{Na}]^{+}$.

Fmoc-Dphg-Dphg-OMe. White solid (88\%), ${ }^{1} \mathrm{H}$ NMR $\delta\left(400 \mathrm{MHz}, \mathrm{CDCl}_{3}\right): 3.78$ (s, 3H), 4.18 $(\mathrm{t}, 1 \mathrm{H}, \mathrm{J}=6.6 \mathrm{~Hz}), 4.49,(\mathrm{~m}, 2 \mathrm{H}), 5.18$ (br s, 1H), 6.6 ( br, s, 1H), 7.26-7.81 (m, 28H). Anal. Calcd. for $\mathrm{C}_{44} \mathrm{H}_{36} \mathrm{~N}_{2} \mathrm{O}_{5}$ (672.77): C, 78.55; H, 5.39; N, 4.16. Found: C, 78.51; H, 5.32; N, 4.04. ES MS: $\mathrm{m} / \mathrm{z}=696.3[\mathrm{M}+\mathrm{Na}]^{+}$.

Fmoc-Dphg-Aib-OBzl. White solid $(90 \%),{ }^{1} \mathrm{H}$ NMR $\delta\left(400 \mathrm{MHz}, \mathrm{CDCl}_{3}\right): 1.53(\mathrm{~s}, 6 \mathrm{H}), 4.18(\mathrm{t}$, $\mathrm{J}=6.5 \mathrm{~Hz}, 1 \mathrm{H}), 4.45(\mathrm{~m}, \mathrm{~J}=6.6 \mathrm{~Hz}, 2 \mathrm{H}), 5.14$ ( s, 2H), 5.8 (br s, 1H), 6.3 (br, 1H), 7.2 - 7.81 (m, 23H). Anal. Calcd. for $\mathrm{C}_{40} \mathrm{H}_{36} \mathrm{~N}_{2} \mathrm{O}_{5}$ (624.33): C, 76.88; H, 5.81; N, 4.48. Found: C, 76.69; H, 5.70; $\mathrm{N}, 4.33$. ES MS: $\mathrm{m} / \mathrm{z}=647.1[\mathrm{M}+\mathrm{Na}]^{+}$.

\section{Acknowledgements}

We thank CSIR, Govt. of India for financial support. One of the authors (RVRR) is grateful to the CSIR for award of a fellowship. The authors also thank Professor Fred Naider, City University of New York, USA for useful discussions. 


\section{References}

1. (a) Carpino, L. A.; Beyermann, M.; Wensehuh. H.; Bienert, M. Acc. Chem. Res. 1996, 29, 268. (b) Hruby, V. J. Acc. Chem. Res. 2001, 34, 389. (c) Humphrey, J. M.; Chamberlin. A. R. Chem. Rev. 1997, 97, 2243. (d) Sambasivarao, K. Acc. Chem. Res. 2003, 36, 342.

2. Beyermann, M.; Bienert, M.; Carpino, L. Houben-Weyl methods of organic chemistry, synthesis of peptides and peptidomimetics; Goodman, E.; Felix, A.; Moroder, L.; Toniolo, C., Eds., Thieme: New York, 2002, E 22a, pp 475-493.

3. Carpino, L. A.; Cohen, B. J.; Kenton, E.; Stephen, Jr. S.; Sadat-Aalee, S. Y.; Tien, J. H.; Lengredge, D. C. J. Org. Chem. 1986, 51, 3732.

4. Tung, R. D.; Dhaon, M. K.; Rich, D. H. J. Org. Chem. 1986, 51, 3350.

5. Freidinger, R. M.; Williams, P. D.; Tung, R. D.; Bock, M. G.; Pettibone, D. J.; Clineschmist, B. V.; Di Pardo, R. M.; Erb, J. M.; Garsky, V. M.; Golud, N. P.; Kaufman, M. J.; Lundell, G. F.; Perlow, D. S.; Whitter, W. L.; Veber, D. F. J. Med. Chem. 1990, 33, 1843.

6. Sivanandaiah, K. M.; Suresh Babu, V. V.; Shankaramma, S. C.; Int. J. Peptide Protein Res. 1994, 44, 24.

7. Suresh Babu, V. V.; Gopi, H. N. Tetrahedron Lett. 1998, 39, 1049.

8. Gopi, H. N.; Suresh Babu, V. V. Ind. J. Chem. 1998, 37B, 394.

9. Tantry, S. J.; Suresh Babu, V. V. Lett. Pept. Sci. 2002, 9, 35.

10. Gopi, H. N.; Suresh Babu, V. V. Tetrahedron Lett. 1998, 39, 9769.

11. Cho, D. H.; Jang, D. O. Tetrahedron Lett. 2004, 45, 2285.

12. Perreux, L.; Loupy, A. Tetrahedron, 2001, 57, 9199.

13. Lidstrom, P.; Tierney, J.; Wathey, B.; Westman, J. Tetrahedron, 2001, 57, 9225.

14. Das, S. K. Synlett 2004, 915.

15. Vasanthakumar, G. R.; Patil, B. S.; Suresh Babu, V. V. Lett. Pept. Sci. 2002, 9, 207.

16. Patil, B. S.; Vasanthakumar, G. R.; Suresh Babu, V. V. Lett. Pept. Sci. 2002, 9, 231.

17. Tantry, S. J.; Kanthararu.; Suresh Babu, V. V. Tetrahedron Lett. 2002, 43, 9461.

18. Patil, B. S.; Vasanthakumar, G. R.; Suresh Babu, V. V. J. Org. Chem. 2003, 68, 7274.

19. Suresh Babu, V. V. Synthesis of peptides mediated by bismuth powder. unpublished results.

20. Yu, H. M.; Chen, S. T.; Wang, K.T. J. Org. Chem. 1992, 57, 4781.

21. Erdelyi, M.; Gogoll. A. Synthesis, 2002, 1592.

22. Murrey, J. K.; Gellman, S. H. Org. Lett. 2005, 7, 1517.

23. Santagada, V.; Fiorino, F.; Perissutti, E.; Severino, B.; De-Filippis, V.; Vivenzio, B.; Calieendo, G. Tetrahedron Lett. 2001, 42, 5171.

24. Urry, D. W.; Cunningham, W. D.; Ohnishi, T. Biochemistry, 1974, 13, 609. 